\title{
INFLUÊNCIA DA SATURAÇÃO POR BASES DO SOLO SOBRE A DISPONIBILIDADE E ABSORÇÃO DE ZINCO
}

\author{
Felipe Augusto Reis Gonçalves ${ }^{1 *}$, Felipe Oliveira Xavier ${ }^{1}$, Rodrigo Rocha Silva $^{2}$, Leonardo \\ Angelo de Aquino ${ }^{3}$, André Mundstock Xavier de Carvalho ${ }^{3}$, Roberto Ferreira Novais ${ }^{4}$ \\ ${ }^{1}$ M.Sc. Engenheiro Agrônomo, Universidade Federal de Viçosa Campus Rio Paranaíba - MG. *E-mail: \\ felipe.a.agro@gmail.com \\ ${ }^{2}$ Engenheiro Agrônomo pela Rizosfera, Mestrando, Universidade Federal de Viçosa Campus Rio Paranaíba - \\ MG. \\ ${ }^{3}$ Professor Adjunto IV e III, respectivamente, Universidade Federal de Viçosa Campus Rio Paranaíba - MG. \\ ${ }^{4}$ Professor Convidado, Universidade Federal de Viçosa Campus Rio Paranaíba - MG.
}

RESUMO: A saturação por base do solo pode influenciar a disponibilidade de zinco para as plantas. Portanto, objetivou-se avaliar a influência de doses de zinco e de crescentes saturações por bases de solo sobre a disponibilidade deste para plantas de milho. Para tal conduziu-se dois experimentos em casa de vegetação, sendo um referente a um solo arenoso e outro argiloso. Ambos em esquema fatorial 6 x 3, em delineamento inteiramente casualizados, com três repetições. O primeiro fator correspondeu a saturação por bases (V\%) de cada solo, após curva de incubação com doses crescentes de calcário, e o segundo fator às doses estudadas de zinco $\left(0,5\right.$ e $\left.25 \mathrm{mg} \mathrm{dm}^{-3}\right)$. Avaliou-se a produção de matéria seca da parte área de plantas de milho, o teor e o acúmulo foliar de zinco, e o teor de zinco no solo após a condução dos experimentos. O aumento da dose de zinco proporcionou aumento de todas variáveis em estudos, em ambos experimentos, exceto para a matéria seca no solo arenoso. Esta, também, foi a única situação que os fatores atuaram de forma independente. $\mathrm{O}$ aumento da saturação por bases do solo proporcionou decréscimo das variáveis analisadas, sendo que o tipo de solo influencia na amplitude da resposta. Concluise que o aumento da dose de zinco contribui para aumento da disponibilidade e acúmulo de zinco para as plantas, sendo esta é afetada negativamente pelo aumento da saturação por bases do solo.

Palavras-chave: Calcário. Sulfato de zinco. Teor foliar. Zea mays.

\section{INFLUENCE OF SATURATION BY SOIL BASES ON THE AVAILABILITY AND ABSORPTION OF ZINC}

\begin{abstract}
Soil saturation can influence the availability of zinc to the plants. Therefore, the objective of this study was to evaluate the influence of zinc doses and increasing soil base saturations on corn availability. For that, two experiments were carried out in a greenhouse, being one referring to a sandy soil and another clayey soil. Both in a $6 \times 3$ factorial scheme, in a completely randomized design, with three replications. The first factor corresponded to the base saturation (V\%) of each soil, after incubation curve with
\end{abstract}


increasing doses of limestone, the second factor at the doses of zinc $\left(0,5\right.$ and $\left.25 \mathrm{mg} \mathrm{dm}^{-3}\right)$. The dry matter production of the corn area area, leaf content and zinc accumulation, and zinc content in the soil were evaluated after conducting the experiments. Increasing the zinc dose gave rise to all variables in studies, in both experiments, except for the dry matter in the sandy soil. This, too, was the only situation where the factors acted independently. The increase of the base saturation of the soil provided a decrease of the variables analyzed, being that the type of soil influences the amplitude of the response. It is concluded that the increase of the zinc dose contributes to an increase in the availability and accumulation of zinc for the plants, which is negatively affected by the increase of the base saturation of the soil.

Key words: Limestone. Zinc sulfate. Foliar contente. Zea mays.

\section{INTRODUÇÃO}

O milho é um cereal altamente responsivo a fertilização com zinco (Zn) (GALRÃO e MESQUITA FILHO, 1981), sendo o micronutriente que propicia maior potencial de resposta à adubação na produtividade de grãos do milho. No entanto, o Zn torna-se limitante para grande parte das culturas, devido sua baixa concentração natural no solo e suas interações com a frações mineral e orgânica. Parte dele encontra-se adsorvido nas partículas de argila goethita, cerca de 30 a $60 \%$ do total, e parte imobilizada ou complexada na matéria orgânica do solo (ARAÚJO e SILVA, 2012). Essas interações são dependentes de CTC, pH e mineralogia do solo, ocorrendo, geralmente, maior adsorção em solos argilosos e menor em solos arenosos (OLIVEIRA et al., 1999; ROSOLEM e FRANCO, 2000).

Na região do Alto Paranaíba-MG, é comum o cultivo do milho em rotação com culturas olerícolas. Nestas, são utilizadas altas doses de fertilizantes e calcários, e o Zn está presente tanto nas formulações de semeadura das hortaliças, bem como contaminante de rochas calcárias (OLIVEIRA et al., 2009). Assim, é comum os solos da região possuírem alto teor de $\mathrm{Zn}$ mesmo com valores elevados de $\mathrm{pH}$ (DEZORDI et al., 2015). Ainda assim, o $\mathrm{Zn}$ aparece como o nutriente com maior limitação na produtividade do milho por deficiência nutricional (GOTT et al., 2016).

A aplicação de micronutrientes pode ser tanto via solo quanto via foliar. Micronutrientes catiônicos aplicados via solo têm sua eficiência reduzida, principalmente em solos de elevado pH. Segundo Valares et al. (2014), em solos com pH superior a 7, há redução da disponibilidade destes micronutrientes. Nessas condições, o Zn no solo, passa de formas solúveis para formas não solúveis.

Objetivou-se avaliar o efeito da saturação por bases do solo e de doses de zinco sobre a disponibilidade deste e o crescimento inicial do milho.

Cultura Agronômica, Ilha Solteira, v.27, n.2, p.262-272, 2018 


\section{MATERIAL E MÉTODOS}

Foram conduzidos dois experimentos em casa de vegetação, na Universidade Federal de Viçosa Campus Rio Paranaíba. Cada experimento correspondeu a um tipo de solo, sendo um Latossolo vermelho textura muito argilosa (solo argiloso) e um Neossolo quartzarênico (solo arenoso). Antes da aplicação dos tratamentos o solo argiloso apresentava: $\mathrm{pH}$ (em $\left.\mathrm{H}_{2} \mathrm{O}\right)=4,5 ; \mathrm{P}$-remanescente $=12,0 \mathrm{mg} \mathrm{L}^{-1} ; \mathrm{P}=0,6 \mathrm{mg} \mathrm{dm}^{-3} ; \mathrm{K}^{+}=16 \mathrm{mg} \mathrm{dm}^{-3} ; \mathrm{Ca}^{2+}=1,7$ $\mathrm{cmol}_{\mathrm{c}} \mathrm{dm}^{-3} ; \mathrm{Mg}^{2+}=0,17 \mathrm{cmol}_{\mathrm{c}} \mathrm{dm}^{-3} ; \mathrm{Al}^{3+}=0,0 \mathrm{cmol}_{\mathrm{c}} \mathrm{dm}^{-3} ; \mathrm{H}+\mathrm{Al}=6,8 \mathrm{cmol}_{\mathrm{c}} \mathrm{dm}^{-3} ; \mathrm{S}=21,3$ $\mathrm{mg} \mathrm{dm}{ }^{-3} ; \mathrm{B}=0,8 \mathrm{mg} \mathrm{dm}^{-3} ; \mathrm{Cu}=1,3 \mathrm{mg} \mathrm{dm}^{-3} ; \mathrm{Fe}=48,2 \mathrm{mg} \mathrm{dm}^{-3} ; \mathrm{Mn}=7,3 \mathrm{mg} \mathrm{dm}^{-3} ; \mathrm{Zn}=$ $0,5 \mathrm{mg} \mathrm{dm}^{-3} ; \mathrm{M} . \mathrm{O}=5,2 \mathrm{~g} \mathrm{~kg}^{-1} \mathrm{e}$ o solo arenoso: $\mathrm{pH}\left(\mathrm{em} \mathrm{H}_{2} \mathrm{O}\right)=4,0$; P-remanescente $=39,4$ $\mathrm{mg} \mathrm{L}{ }^{-1} ; \mathrm{P}=6,6 \mathrm{mg} \mathrm{dm}^{-3} ; \mathrm{K}^{+}=42 \mathrm{mg} \mathrm{dm}^{-3} ; \mathrm{Ca}^{2+}=0,4 \mathrm{cmol}_{\mathrm{c}} \mathrm{dm}^{-3} ; \mathrm{Mg}^{2+}=0,2 \mathrm{cmol}_{\mathrm{c}} \mathrm{dm}^{-3}$; $\mathrm{Al}^{3+}=0,5 \mathrm{cmol}_{\mathrm{c}} \mathrm{dm}^{-3} ; \mathrm{H}+\mathrm{Al}=4,3 \mathrm{cmol}_{\mathrm{c}} \mathrm{dm}^{-3} ; \mathrm{S}=22,2 \mathrm{mg} \mathrm{dm}^{-3} ; \mathrm{B}=1,0 \mathrm{mg} \mathrm{dm}^{-3} ; \mathrm{Cu}=$ $0,16 \mathrm{mg} \mathrm{dm}^{-3} ; \mathrm{Fe}=55,9 \mathrm{mg} \mathrm{dm}^{-3} ; \mathrm{Mn}=4,2 \mathrm{mg} \mathrm{dm}^{-3} ; \mathrm{Zn}=1,12 \mathrm{mg} \mathrm{dm}^{-3} ; \mathrm{M} \cdot \mathrm{O}=2,3 \mathrm{~g} \mathrm{~kg}^{-1}$. As análises foram realizadas segundo metodologia descrita por Donagema et al. (2011).

Foi utilizado o delineamento experimental inteiramente casualizado, com três repetições. Cada experimento foi esquematizado em um fatorial $6 \times 3$, onde o primeiro fator correspondeu a saturação por bases (V\%) esperada de cada solo e o segundo fator às doses estudadas de zinco. Para obtenção das saturações esperadas, procedeu-se a incubação de ambos os solos com doses crescentes de calcário. Para o solo argiloso obteve-se as saturações de 21,$5 ; 34,0 ; 51,0 ; 58,0 ; 61,0 ; 65,6 \%$, sendo o $\mathrm{pH}$ correspondente a cada saturação igual à 4,52;4,86;5,45;5,87; 6,29 e 6,49 , respectivamente. No solo arenoso as saturações obtidas foram iguais 10,$5 ; 34,0 ; 49,0 ; 58,0 ; 61,0 ; 61,5 \%$, e pH correspondente de 3,$99 ; 4,59 ; 5,54 ; 6,41 ; 6,77$ e 7,15, respectivamente. As doses de zinco foram iguais a $0,5 \mathrm{e}$ $25 \mathrm{mg} \mathrm{dm}^{-3}$ de $\mathrm{Zn}$, sendo aplicadas via sulfato de zinco p.a. (22,60 \% de Zn). Após a homogeneização de cada solo com o zinco, procedeu-se a incubação durante quinze dias, ambos com sua respectiva capacidade de campo.

Semeou-se o milho híbrido RB 9110 VT PRO 2, em vasos de $4 \mathrm{dm}^{3}$, sendo deixadas quatro plantas por vaso após o desbaste. A adubação foi composta de $150 \mathrm{mg} \mathrm{dm}^{-3} \mathrm{de} \mathrm{N}$, $300 \mathrm{mg} \mathrm{dm}^{-3}$ de P, $180 \mathrm{mg} \mathrm{dm}^{-3}$ de K, $50 \mathrm{mg} \mathrm{dm}^{-3}$ de S e $0,5 \mathrm{mg} \mathrm{dm}^{-3}$ de B. Sendo o $\mathrm{N}$ e o K aplicados na quantidade de 15 e $75 \mathrm{mg} \mathrm{dm}^{-3}$, respectivamente, no dia da semeadura e o restante parcelado em cobertura. Cada vaso de $4 \mathrm{dm}^{3}$ com quatro plantas representou uma unidade experimental.

Aos 40 dias após a emergência das plântulas procedeu-se a determinação da matéria seca, do teor foliar e acúmulo de $\mathrm{Zn}$. Para tal, todas plantas de cada vaso foram coletadas e levadas para estufa de circulação forçada de ar a $70{ }^{\circ} \mathrm{C}$ por $72 \mathrm{~h}$. Posteriormente foram trituradas em moinho tipo Willey e levadas para laboratório para determinação de teor foliar de zinco, segundo métodos descritos em Malavolta, Vitti e Oliveira (1997). O acúmulo foi calculado pelo produto entre a matéria seca e o teor. Uma amostra de solo de $100 \mathrm{~g}$ foi coletada em cada vaso a fim de determinar o teor de zinco no solo após os experimentos, segundo metodologia de solução mista de ácidos (DONAGEMA et al., 2011).

Cultura Agronômica, Ilha Solteira, v.27, n.2, p.262-272, 2018 
O fatorial foi submetido à análise de variância. Quando significativo, realizou-se o desdobramento dos graus de liberdades das interações. As doses de zinco dentro de cada saturação foram comparadas pelo teste de Student-Newman-Keuls a $5 \%$ de probabilidade e realizada análise de regressão para as saturações.

\section{RESULTADOS E DISCUSSÃO}

A matéria seca do milho cultivado em solo arenoso foi superior na ausência de fertilização com zinco (Tabela 1). Ambas as doses aplicadas (5 e $25 \mathrm{mg} \mathrm{dm}^{-3}$ ) proporcionaram uma redução na matéria seca das plantas possivelmente por serem fitotóxicas a elas. O solo arenoso, por suas características mineralógicas, como baixo teor de argila, proporciona uma baixa adsorção de $\mathrm{Zn}$, que fica em quase totalidade na solução do solo e propensa a absorção pela planta. Com isso, mesmo a menor dose utilizada ultrapassou a quantidade requerida pela cultura, sendo tóxica e reduzindo o crescimento inicial do milho, independente da saturação por bases do solo. Steiner et al. (2011) não verificaram diferenças na altura de plantas e parâmetros relacionados ao crescimento do milho entre as doses 0 e $5 \mathrm{mg} \mathrm{dm}^{-3}$ em solo com $52 \%$ de saturação por bases.

Tabela 1. Matéria seca, teor e acúmulo foliar de zinco em plantas de milho cultivadas em solo arenoso submetido a diferentes saturações de bases (V\%) e doses de zinco.

\begin{tabular}{|c|c|c|c|c|c|}
\hline \multirow{3}{*}{$\begin{array}{c}\text { Saturação } \\
(\%)\end{array}$} & \multirow{3}{*}{$\mathrm{pH}$} & \multicolumn{3}{|c|}{ Dose de $\mathrm{Zn}\left(\mathrm{mg} \mathrm{dm}^{-3}\right)$} & \multirow{3}{*}{ Média } \\
\hline & & 0 & 5 & 25 & \\
\hline & & \multicolumn{3}{|c|}{ Matéria seca $\left(\mathrm{g} \mathrm{vaso}^{-1}\right)$} & \\
\hline 10,5 & 3,99 & 7,64 & 7,17 & 6,45 & $7,09 \mathrm{c}$ \\
\hline 34,0 & 4,59 & 9,87 & 8,35 & 8,09 & $8,77 \mathrm{a}$ \\
\hline 49,0 & 5,54 & 9,01 & 8,32 & 8,72 & $8,69 \mathrm{a}$ \\
\hline 58,0 & 6,41 & 8,72 & 7,79 & 8,99 & $8,50 \mathrm{ab}$ \\
\hline 61,0 & 6,77 & 7,91 & 7,64 & 7,66 & $7,74 \mathrm{bc}$ \\
\hline 61,5 & 7,15 & 8,59 & 7,51 & 7,62 & $7,91 \mathrm{ab}$ \\
\hline \multirow[t]{2}{*}{ Média } & & $8,62 \mathrm{~A}$ & $7,80 \mathrm{~B}$ & $7,92 \mathrm{~B}$ & \\
\hline & & $\mathrm{F}_{\mathrm{S}}=8,18^{* *}$ & $\mathrm{~F}_{\mathrm{D}}=7,55^{* *}$ & $\mathrm{~F}_{\mathrm{S} * \mathrm{D}}=1,25^{\mathrm{ns}}$ & $\mathrm{CV}(\%)=8,50$ \\
\hline \multicolumn{6}{|c|}{ Teor foliar de zinco $\left(\mathrm{mg} \mathrm{kg}^{-1}\right)$} \\
\hline 10,5 & 3,99 & $22,42 \mathrm{C}$ & $114,53 \mathrm{~B}$ & $149,90 \mathrm{~A}$ & 149,90 \\
\hline 34,0 & 4,59 & $18,50 \mathrm{C}$ & $84,77 \mathrm{~B}$ & $202,34 \mathrm{~A}$ & 101,87 \\
\hline 49,0 & $5,54 \$$ & $13,55 \mathrm{C}$ & $57,59 \mathrm{~B}$ & $137,02 \mathrm{~A}$ & 69,38 \\
\hline 58,0 & 6,41 & $10,38 \mathrm{C}$ & $42,93 \mathrm{~B}$ & $87,25 \mathrm{~A}$ & 46,85 \\
\hline 61,0 & 6,77 & $10,07 \mathrm{C}$ & $45,43 \mathrm{~B}$ & $94,16 \mathrm{~A}$ & 49,89 \\
\hline 61,5 & 7,15 & $8,96 \mathrm{C}$ & $42,38 \mathrm{~B}$ & $94,26 \mathrm{~A}$ & 48,54 \\
\hline \multirow[t]{2}{*}{ Média } & & $13,98 \mathrm{C}$ & $64,61 \mathrm{~B}$ & $154,63 \mathrm{~A}$ & \\
\hline & & $\mathrm{F}_{\mathrm{S}}=414,19 * *$ & $\mathrm{~F}_{\mathrm{D}}=2489,9 * *$ & $\mathrm{~F}_{\mathrm{S} * \mathrm{D}}=151,52 * *$ & $\mathrm{CV}(\%)=7,79$ \\
\hline
\end{tabular}


Tabela 1. Continuação...

\begin{tabular}{|c|c|c|c|c|c|}
\hline \multicolumn{6}{|c|}{ Acúmulo foliar de zinco $\left(\mathrm{mg} \mathrm{vaso}^{-1}\right)$} \\
\hline 10,5 & 3,99 & $0,17 \mathrm{C}$ & $1,18 \mathrm{~B}$ & $2,91 \mathrm{~A}$ & 1,42 \\
\hline 34,0 & 4,59 & $0,16 \mathrm{C}$ & $0,72 \mathrm{~B}$ & $1,59 \mathrm{~A}$ & 0,82 \\
\hline 49,0 & 5,54 & $0,10 \mathrm{C}$ & $0,46 \mathrm{~B}$ & $1,08 \mathrm{~A}$ & 0,55 \\
\hline 58,0 & 6,41 & $0,08 \mathrm{C}$ & $0,33 \mathrm{~B}$ & $0,66 \mathrm{~A}$ & 0,35 \\
\hline 61,0 & 6,77 & $0,07 \mathrm{C}$ & $0,37 \mathrm{~B}$ & $1,01 \mathrm{~A}$ & 0,48 \\
\hline 61,5 & 7,15 & $0,08 \mathrm{C}$ & $0,33 \mathrm{~B}$ & $0,72 \mathrm{~A}$ & 0,37 \\
\hline \multirow{2}{*}{ Média } & & $0,11 \mathrm{C}$ & $0,56 \mathrm{~B}$ & $1,33 \mathrm{~A}$ & \\
\hline & & $\mathrm{F}_{\mathrm{S}}=701,08^{* * *}$ & $\mathrm{~F}_{\mathrm{D}}=3946,33^{* *}$ & $\mathrm{~F}_{\mathrm{S} * \mathrm{D}}=357,97^{* *}$ & $\mathrm{CV}(\%)=6,06$ \\
\hline
\end{tabular}

Médias seguidas pela mesma letra maiúscula na linha não diferem entre si pelo teste de Student-Newman-Keauls a $5 \%$ de probabilidade. ${ }^{\text {ns }}$ não significativo $(\mathrm{p}>=0,05)$.* significativo ao nível de $5 \%$ de probabilidade $(0,01=<\mathrm{p}<0,05)$. ** significativo ao nível de $1 \%$ de probabilidade $(\mathrm{p}<0,01)$. $\mathrm{F}_{\mathrm{s}}$ : $\mathrm{F}$ calculado para fator saturação. $\mathrm{F}_{\mathrm{D}}$ : $\mathrm{F}$ calculado para fator dose. FF*D: F calculado para interação saturação $\mathrm{x}$ dose.

Já no solo argiloso, houve interação entre dose de Zn e saturação por bases. No solo com menor V\%, a maior dose de $\mathrm{Zn}\left(25 \mathrm{mg} \mathrm{dm}^{-3}\right)$ reduziu a matéria seca (Tabela 2). Com o aumento da saturação por bases, a fertilização com $\mathrm{Zn}$ aumentou os valores desta variável. Nestes solos, há maior adsorção de Zn no solo, e como há o aumento do pH com aumento da $\mathrm{V} \%$, há grande indisponibilidade de Zn às plantas por sua precipitação no solo. Assim, solos com elevada saturação necessitam de ser suplementadas com $\mathrm{Zn}$ para um maior crescimento do milho. Assim, a ausência no suprimento deste nutriente em solos de elevada saturação não proporciona a obtenção do máximo crescimento inicial da cultura. No entanto, Pereira, Ernani e Sangoi (2007) não verificaram incrementos na matéria seca do milho mesmo com a adição de calcário, e a consequente elevação do $\mathrm{pH}$, em quatro cultivos sucessivos.

A saturação por bases influenciou a matéria seca da parte aérea do milho. Para o solo arenoso, o modelo quadrático foi o que melhor se ajustou ao comportamento da matéria seca em detrimento do V\% real do solo (Figura 1A). Houve incrementos na matéria seca até V\% em torno de $50 \%$. Esta saturação é a recomendada para a cultura do milho (ALVAREZ V. e RIBEIRO, 1999). Acima deste valor, como efeito indireto, têm-se o aumento do $\mathrm{pH}$, que indisponibiliza os micronutrientes catiônicos, como o $\mathrm{Zn}$, o que pode limitar o crescimento da planta (VALADARES et al., 2014). Já para o solo argiloso, na ausência da adubação de $\mathrm{Zn}$, houve redução na matéria seca com o aumento da saturação. Figueiredo et al. (2012) verificaram incremento quadrático, entre 27 e $62 \%$ de saturação por bases, em um solo argiloso, na matéria seca do milho. Com a aplicação de Zn no solo, nas duas doses, não houve efeito da saturação na matéria seca do milho. A redução na matéria secado milho somente na ausência da adubação com Zn, é explicada pelo efeito da saturação na indisponibilização por precipitação deste nutriente à planta, ao passo que este efeito é minimizado na presença de $\mathrm{Zn}$ no solo.

Cultura Agronômica, Ilha Solteira, v.27, n.2, p.262-272, 2018 
Tabela 2. Matéria seca de plantas de milho cultivadas em solo argiloso com submetido a diferentes saturações de bases (V\%) e doses de zinco.

\begin{tabular}{|c|c|c|c|c|c|}
\hline \multirow{3}{*}{$\begin{array}{c}\text { Saturação } \\
(\%)\end{array}$} & \multirow{3}{*}{$\mathrm{pH}$} & \multicolumn{3}{|c|}{ Dose de $\mathrm{Zn}\left(\mathrm{mg} \mathrm{dm}^{-3}\right)$} & \multirow{3}{*}{ Média } \\
\hline & & 0 & 5 & 25 & \\
\hline & & \multicolumn{3}{|c|}{ Matéria seca $\left(\right.$ g vaso $\left.^{-1}\right)$} & \\
\hline 21,5 & 4,52 & $10,91 \mathrm{~A}$ & $10,79 \mathrm{~A}$ & $8,20 \mathrm{~B}$ & 9,97 \\
\hline 34,0 & 4,86 & $9,57 \mathrm{~A}$ & $9,22 \mathrm{~A}$ & $9,11 \mathrm{~A}$ & 9,30 \\
\hline 51,0 & 5,45 & $5,75 \mathrm{~A}$ & $7,30 \mathrm{~A}$ & $7,99 \mathrm{~A}$ & 7,02 \\
\hline 58,0 & 5,87 & $5,61 \mathrm{~B}$ & $8,90 \mathrm{~A}$ & $9,06 \mathrm{~A}$ & 7,85 \\
\hline 61,0 & 6,29 & 4,94 B & $9,39 \mathrm{~A}$ & $8,49 \mathrm{~A}$ & 7,60 \\
\hline 65,6 & 6,49 & $3,37 \mathrm{C}$ & $9,24 \mathrm{~A}$ & $6,97 \mathrm{~B}$ & 6,53 \\
\hline \multirow[t]{2}{*}{ Média } & & $6,69 \mathrm{~B}$ & $9,14 \mathrm{~A}$ & $8,30 \mathrm{~A}$ & \\
\hline & & $\mathrm{F}_{\mathrm{S}}=9,94^{* *}$ & $\mathrm{~F}_{\mathrm{D}}=17,41^{* *}$ & $\mathrm{~F}_{\mathrm{S} * \mathrm{D}}=4,67^{* *}$ & $\mathrm{CV}(\%)=15,74$ \\
\hline \multicolumn{6}{|c|}{ Teor foliar de zinco $\left(\mathrm{mg} \mathrm{kg}^{-1}\right)$} \\
\hline 21,5 & 4,52 & $7,97 \mathrm{C}$ & $32,80 \mathrm{~B}$ & $171,55 \mathrm{~A}$ & 70,77 \\
\hline 34,0 & 4,86 & $7,88 \mathrm{C}$ & $33,19 \mathrm{~B}$ & $116,07 \mathrm{~A}$ & 52,38 \\
\hline 51,0 & 5,45 & $11,40 \mathrm{C}$ & $30,13 \mathrm{~B}$ & $66,89 \mathrm{~A}$ & 34,16 \\
\hline 58,0 & 5,87 & $8,96 \mathrm{C}$ & $26,48 \mathrm{~B}$ & $62,00 \mathrm{~A}$ & 32,48 \\
\hline 61,0 & 6,29 & $8,53 \mathrm{C}$ & $22,39 \mathrm{~B}$ & $57,27 \mathrm{~A}$ & 29,40 \\
\hline 65,6 & 6,49 & $7,81 \mathrm{C}$ & $22,14 \mathrm{~B}$ & $52,46 \mathrm{~A}$ & 27,47 \\
\hline \multirow[t]{2}{*}{ Média } & & $8,76 \mathrm{C}$ & $27,86 \mathrm{~B}$ & $87,70 \mathrm{~A}$ & \\
\hline & & $\mathrm{F}_{\mathrm{S}}=180,69^{* *}$ & $\mathrm{~F}_{\mathrm{D}}=2145,84^{* *}$ & $\mathrm{~F}_{\mathrm{S} * \mathrm{D}}=146,38^{* *}$ & $\mathrm{CV}(\%)=9,10$ \\
\hline \multicolumn{6}{|c|}{ Acúmulo foliar de zinco $\left(\mathrm{mg} \mathrm{vaso}^{-1}\right)$} \\
\hline 21,5 & 4,52 & $0,09 \mathrm{C}$ & $0,33 \mathrm{~B}$ & $0,78 \mathrm{~A}$ & 0,40 \\
\hline 34,0 & 4,86 & $0,04 \mathrm{C}$ & $0,17 \mathrm{~B}$ & $0,30 \mathrm{~A}$ & 0,17 \\
\hline 51,0 & 5,45 & $0,11 \mathrm{C}$ & $0,28 \mathrm{~B}$ & $0,51 \mathrm{~A}$ & 0,30 \\
\hline 58,0 & 5,87 & $0,08 \mathrm{C}$ & $0,25 \mathrm{~B}$ & $0,57 \mathrm{~A}$ & 0,30 \\
\hline 61,0 & 6,29 & $0,07 \mathrm{C}$ & $0,20 \mathrm{~B}$ & $0,51 \mathrm{~A}$ & 0,26 \\
\hline 65,6 & 6,49 & $0,06 \mathrm{C}$ & $0,19 \mathrm{~B}$ & $0,34 \mathrm{~A}$ & 0,20 \\
\hline \multirow[t]{2}{*}{ Média } & & $0,08 \mathrm{C}$ & $0,24 \mathrm{~B}$ & $0,50 \mathrm{~A}$ & \\
\hline & & $\mathrm{F}_{\mathrm{S}}=67,97^{* *}$ & $\mathrm{~F}_{\mathrm{D}}=928,16^{* *}$ & $\mathrm{~F}_{\mathrm{S} * \mathrm{D}}=22,62^{* *}$ & $\mathrm{CV}(\%)=11,10$ \\
\hline
\end{tabular}

Médias seguidas pela mesma letra maiúscula na linha não diferem entre si pelo teste de Student-Newman-Keauls a $5 \%$ de probabilidade. ${ }^{\mathrm{ns}}$ não significativo $(\mathrm{p}>=0,05)$.* significativo ao nível de $5 \%$ de probabilidade $(0,01=<\mathrm{p}<0,05)$. ** significativo ao nível de $1 \%$ de probabilidade $(\mathrm{p}<0,01)$. $\mathrm{F}_{\mathrm{s}}$ : F calculado para fator saturação. $\mathrm{F}_{\mathrm{D}}$ : F calculado para fator dose. $\mathrm{F}_{\mathrm{F} * \mathrm{D}}$ : F calculado para interação saturação $\mathrm{x}$ dose. 
Solo Arenoso
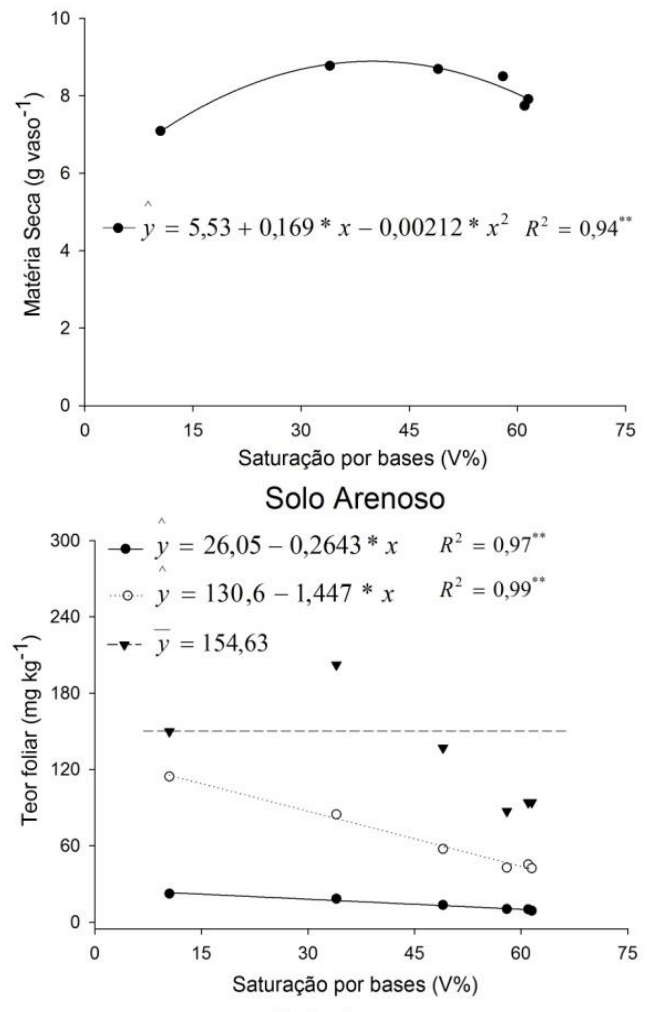

Solo Arenoso

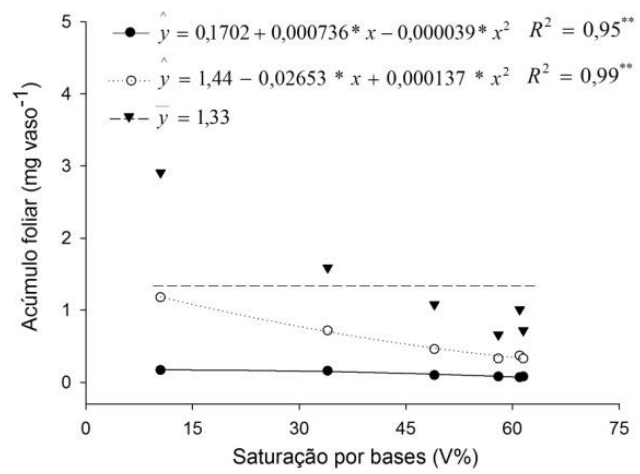

Solo Arenoso

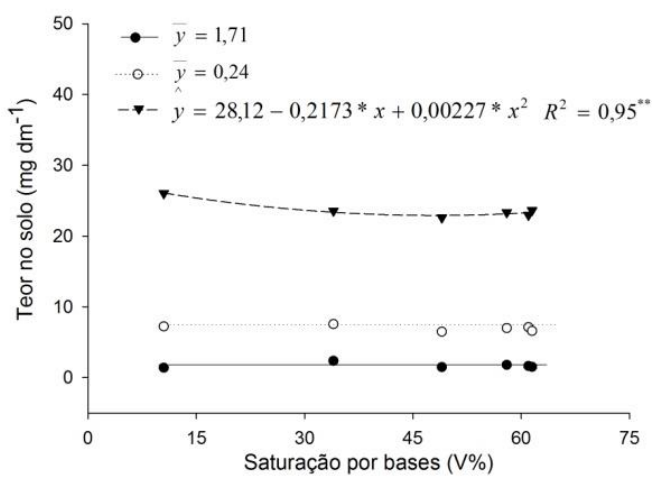

A

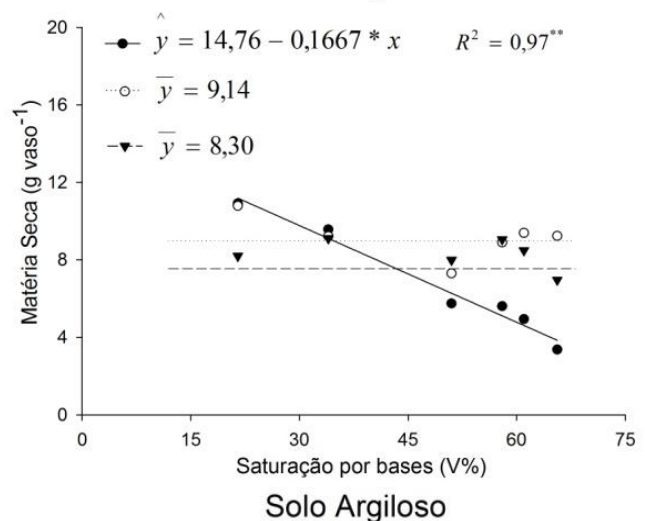

B

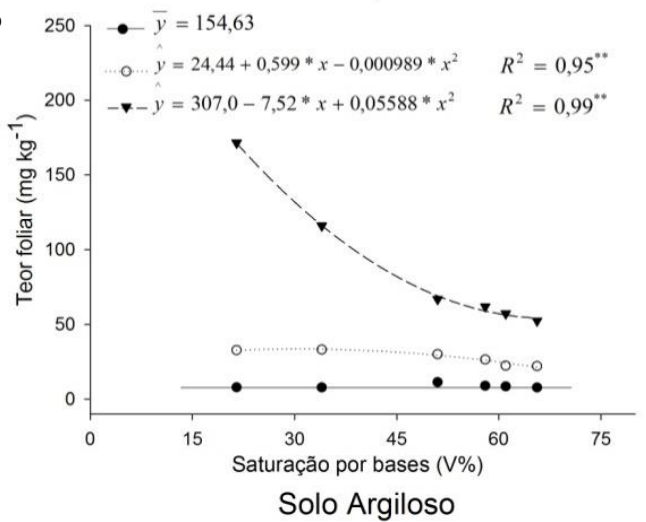

C

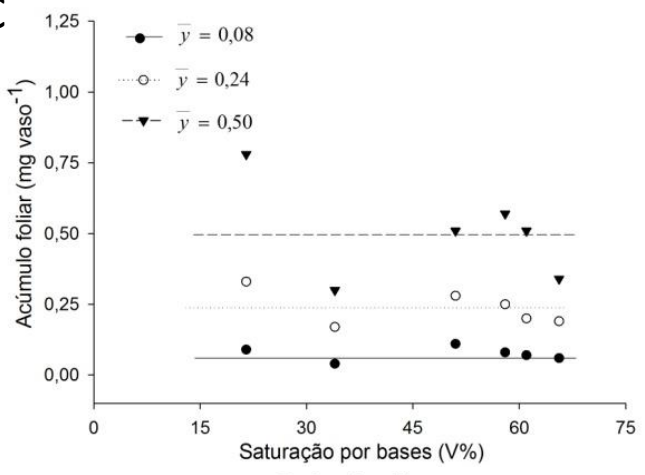

Solo Argiloso

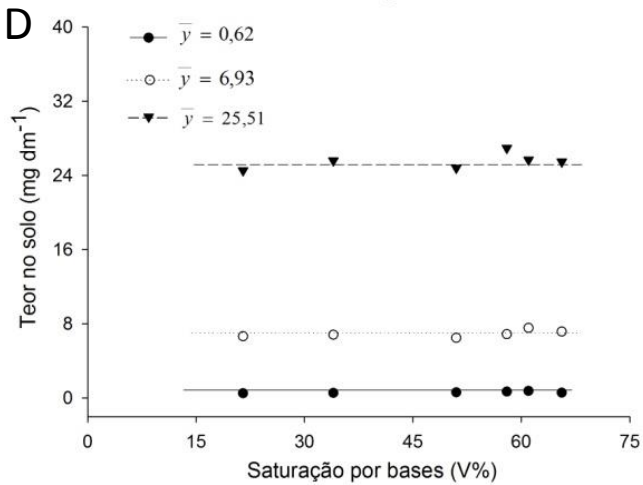

Figura 1. Matéria seca (A), teor foliar (B), acúmulo foliar (C) e teor no solo de zinco (D) em função de diferentes saturações por base $(\mathrm{V} \%)$ em dois tipos de solo (arenoso e argiloso) nas doses de $0(\bullet), 5(\circ)$ e $25(\nabla) \mathrm{mg} \mathrm{dm}^{-3}$.

Cultura Agronômica, Ilha Solteira, v.27, n.2, p.262-272, 2018 
O acúmulo de Zn nas plantas de milho foi influenciado pela interação entre a dose de zinco e a saturação por bases do solo. No entanto, de maneira geral, para os dois solos (arenoso e argiloso), houve incremento no acúmulo com o aumento da dose de Zn no solo, independente do V\% (Tabelas 1 e 2). Resultados similares foram encontrados por Pereira, Ernani e Sangoi (2007), que verificaram incrementos lineares no acúmulo de Zn na parte aérea do milho nas fases iniciais da cultura com aumento da fertilização de $\mathrm{Zn}$ até a dose de $80 \mathrm{mg} \mathrm{dm}^{-3}$. Assim, houve maior absorção e assimilação deste nutriente, quando este foi mais disponível as plantas, mesmo que este acúmulo não tenha representado maior crescimento do milho. Desta forma, pode-se dizer que houve um consumo de luxo pela planta na maior dose de $\mathrm{Zn}$ empregada, no qual planta acumulou quantidades superiores de Zn às necessárias e este pode propiciar uma translocação deste nutriente para outros órgãos posteriormente.

A saturação por bases influenciou no acúmulo de $\mathrm{Zn}$ somente nas doses 0 e $5 \mathrm{mg} \mathrm{dm}^{-}$ ${ }^{3}$, no solo arenoso. Nestas doses, houve um decréscimo no acúmulo com o aumento do V\% do solo, sendo este, ajustado por um modelo quadrático (Figura 1C). Esta tendência no acúmulo também pode ser explicada pela menor disponibilidade do $\mathrm{Zn}$ em solos com elevada saturação, e consequentemente pH. Pereira, Ernani e Sangoi (2007) também verificaram menor disponibilidade e consequentemente acúmulo de $\mathrm{Zn}$ pelo milho com o aumento do $\mathrm{pH}$ do solo pela interação deste com as fases sólidas do mesmo. Para o solo argiloso, não houve ajuste significativo para esta variável entre estes fatores. Assim, não houve influência da saturação no acúmulo para este solo.

O teor de Zn no solo após o cultivo do milho também foi influenciado pela dose de Zn e pela saturação por bases. Como esperado, o aumento da dose de $\mathrm{Zn}$ aplicada proporcionou maior teor deste nutriente na solução do solo, e esta tendência foi observada nos dois solos cultivados (Tabela 3). Pereira, Ernani e Sangoi (2007) também verificaram incrementos no teor de Zn no solo, de forma linear, com a aplicação de $\mathrm{Zn}$ até a dose de $80 \mathrm{mg} \mathrm{dm}^{-3}$. Já a saturação por bases somente influenciou no teor de $\mathrm{Zn}$ no solo, na dose de $25 \mathrm{mg} \mathrm{dm}^{-3}$ no solo arenoso (Figura 1D). Neste, a menor saturação proporcionou maior teor de Zn na solução do solo e as saturações intermediárias reduziram a disponibilidade de Zn, que foi novamente aumentado nos maiores $\mathrm{V} \%$, ficando evidente o ajuste do modelo quadrático. Pereira, Ernani e Sangoi (2007) verificaram maiores quantidades de Zn extraídos em solos com menor $\mathrm{pH}$ em detrimento aos solos que receberam correção da acidez por meio de doses crescentes de calcário. Nas outras doses de Zn do solo arenoso, assim como no solo argiloso, não houve modelos ajustados que explicassem o efeito da saturação por bases do solo sobre o teor de $\mathrm{Zn}$ do mesmo.

Cultura Agronômica, Ilha Solteira, v.27, n.2, p.262-272, 2018 
Tabela 3. Teor de zinco no solo após cultivo de plantas de milho em solo arenoso e argiloso submetidos a diferentes saturações de bases (V\%) e doses de zinco.

\begin{tabular}{|c|c|c|c|c|c|}
\hline \multirow{3}{*}{$\begin{array}{c}\text { Saturação } \\
(\%)\end{array}$} & \multirow{3}{*}{$\mathrm{pH}$} & \multicolumn{3}{|c|}{ Dose de $\mathrm{Zn}\left(\mathrm{mg} \mathrm{dm}^{-3}\right)$} & \multirow{3}{*}{ Média } \\
\hline & & 0 & 5 & 25 & \\
\hline & & \multicolumn{3}{|c|}{ Teor de zinco no solo arenoso $\left(\mathrm{mg} \mathrm{dm}^{-3}\right)$} & \\
\hline 10,5 & 3,99 & $1,41 \mathrm{C}$ & $7,24 \mathrm{~B}$ & $26,04 \mathrm{~A}$ & 11,57 \\
\hline 34,0 & 4,59 & $2,38 \mathrm{C}$ & $7,56 \mathrm{~B}$ & $23,57 \mathrm{~A}$ & 11,17 \\
\hline 49,0 & 5,54 & $1,49 \mathrm{C}$ & $6,49 \mathrm{~B}$ & $22,59 \mathrm{~A}$ & 10,19 \\
\hline 58,0 & 6,41 & $1,81 \mathrm{C}$ & $7,01 \mathrm{~B}$ & $23,35 \mathrm{~A}$ & 10,72 \\
\hline 61,0 & 6,77 & $1,65 \mathrm{C}$ & 7,13 B & $22,97 \mathrm{~A}$ & 10,58 \\
\hline 61,5 & 7,15 & $1,52 \mathrm{C}$ & $6,60 \mathrm{~B}$ & $23,64 \mathrm{~A}$ & 10,59 \\
\hline \multirow[t]{2}{*}{ Média } & & $1,71 \mathrm{C}$ & $7,00 \mathrm{~B}$ & $23,69 \mathrm{~A}$ & \\
\hline & & $\mathrm{F}_{\mathrm{S}}=4,81^{* *}$ & $\mathrm{~F}_{\mathrm{D}}=5294,83^{* *}$ & $\mathrm{~F}_{S^{*} \mathrm{D}}=3,53^{* *}$ & $\mathrm{CV}(\%)=6,19$ \\
\hline \multicolumn{6}{|c|}{ Teor de zinco no solo argiloso $\left(\mathrm{mg} \mathrm{dm}^{-3}\right)$} \\
\hline 21,5 & 4,52 & $0,52 \mathrm{C}$ & $6,66 \mathrm{~B}$ & $24,54 \mathrm{~A}$ & 10,58 \\
\hline 34,0 & 4,86 & $0,56 \mathrm{C}$ & $6,83 \mathrm{~B}$ & $25,60 \mathrm{~A}$ & 11,00 \\
\hline 51,0 & 5,45 & $0,61 \mathrm{C}$ & $6,49 \mathrm{~B}$ & $24,81 \mathrm{~A}$ & 10,64 \\
\hline 58,0 & 5,87 & $0,70 \mathrm{C}$ & $6,88 \mathrm{~B}$ & $26,95 \mathrm{~A}$ & 11,51 \\
\hline 61,0 & 6,29 & $0,77 \mathrm{C}$ & $7,56 \mathrm{~B}$ & $25,69 \mathrm{~A}$ & 11,34 \\
\hline 65,6 & 6,49 & $0,58 \mathrm{C}$ & 7,17 B & $25,48 \mathrm{~A}$ & 11,08 \\
\hline Média & & $0,62 \mathrm{C}$ & $6,93 \mathrm{~B}$ & $25,51 \mathrm{~A}$ & \\
\hline & & $\mathrm{F}_{\mathrm{S}}=6,23^{* *}$ & $\mathrm{~F}_{\mathrm{D}}=15132,7^{* *}$ & $\mathrm{~F}_{\mathrm{S} * \mathrm{D}}=3,38^{* *}$ & $\mathrm{CV}(\%)=4,05$ \\
\hline
\end{tabular}

Médias seguidas pela mesma letra maiúsculas na linha não diferem entre si pelo teste de Student-Newman-Keauls a $5 \%$ de probabilidade. ${ }^{\mathrm{ns}}$ não significativo $(\mathrm{p}>=.05)$.* significativo ao nível de $5 \%$ de probabilidade $(.01=<\mathrm{p}<.05)$. ** significativo ao nível de $1 \%$ de probabilidade $(\mathrm{p}<.01)$. $\mathrm{F}_{\mathrm{s}}$ : F calculado para fator saturação. $\mathrm{F}_{\mathrm{D}}$ : F calculado para fator dose. $\mathrm{F}_{\mathrm{F} * \mathrm{D}}$ : $\mathrm{F}$ calculado para interação saturação $\mathrm{x}$ dose.

\section{CONCLUSÃO}

O aumento da dose de zinco propicia aumento na disponibilidade e acúmulo deste para as plantas.

A saturação por bases do solo afeta negativamente a disponibilidade de zinco, sendo que há influência do tipo de solo quanto ao efeito da saturação.

\section{REFERÊNCIAS BIBLIOGRÁFICAS}

ALVAREZ V., V. H.; RIBEIRO, A. C. Calagem. In: RIBEIRO, A. C.; GUIMARÃES, P. T. G.; ALVAREZ, V. V. H. (Eds.) Recomendações para o uso de corretivos e fertilizantes em Minas Gerais - 5a Aproximação. Viçosa: Comissão de Fertilidade do Solo do Estado de Minas Gerais, 1999. cap. 8, p. 48. 
ARAÚJO, E. O.; SILVA, M. A. C. Interação boro e zinco no crescimento, desenvolvimento e nutrição do algodoeiro. Revista Brasileira de Ciências Agrárias, Pernambuco, v. 7, n. 1, p.720-727, 2012.

DEZORDI, L. R.; CLEMENTE, J. M.; OLIVEIRA, T. F.; GENTIL, T. G.; AQUINO, L. A. Nutrient demand of the carrot crop. African Journal of Agricultural Research, v. 10, n. 35, p.3533-3542, 2015. Disponível em: 〈https://doi.org/10.5897/AJAR2015.9849>. Acesso em: 20 set. 2017.

DONAGEMA, G. K.; DE CAMPOS, D. B.; CALDERANO, S. B.; TEIXEIRA, W. G.; VIANA, J. M. Manual de métodos de análise de solo. Rio de Janeiro: Embrapa Solos, 2011. 225 p. (Documentos INFOTECA-E)

GOTT, R. M.; AQUINO, L. A.; CLEMENTE, J. M.; SANTOS, L. P. D.; CARVALHO, A. M. X.; XAVIER, F. O. Foliar Diagnosis Indexes for Corn by the Methods Diagnosis and Recommendation Integrated System (DRIS) and Nutritional Composition (CND). Communications in Soil Science and Plant Analysis, v. 48, n. 1, p.11-19, 2016. Disponível em: <https://doi.org/10.1080/00103624.2016.1253714>. Acesso em: 20 out. 2017.

FIGUEIREDO, C. C.; BARBOSA, D. V.; OLIVEIRA, S. A.; FAGIOLI, M.; SATO, J. H. Adubo fosfatado revestido com polímero e calagem na produção e parâmetros morfológicos de milho. Revista Ciência Agronômica, Fortaleza, v. 43, n. 3, p.446-452, 2012.

GALRÃO, E. Z.; MESQUITA FILHO, M. V. Efeito de fontes de zinco na produção de matéria seca do milho em um solo sob cerrado. Revista Brasileira de Ciência do Solo, Viçosa, v. 5, n. 3, p.167-170, 1981.

MALAVOLTA, E.; VITTI, G. C.; OLIVEIRA, S. A. Avaliação do estado nutricional das plantas: princípios e aplicações. 2. ed. Piracicaba: Potafos, 1997. 319 p.

OLIVEIRA, M. F. G.; NOVAIS, R. F.; NEVES, J. C. L.; ALVES, V. M. C.; VASCONCELLOS, C. A. Fluxo difusivo de zinco em amostras de solo influenciado por textura, íon acompanhante e pH do solo. Revista Brasileira de Ciência do Solo, Viçosa, v. 23, n. 3, p.609-615, 1999.

OLIVEIRA, R. C.; ARAÚJO, A. R.; CARVALHO, R.; GUILHERME, L. R. G.; PASSOS, L. P.; MARQUES, J. J. Movimento de zinco em colunas de solo tratado com resíduo calcário oriundo de mineração. Revista Ceres, Viçosa, v. 56, n. 5, p.679-684, 2009.

PEREIRA, N. M. Z.; ERNANI, P. R.; SANGOI, L. Disponibilidade de zinco e para o milho afetada pela adição de $\mathrm{Zn}$ e pelo $\mathrm{pH}$ do solo. Revista Brasileira de Milho e Sorgo, Sete Lagoas, v. 6, n. 3, p.273-284, 2007.

ROSOLEM, C. A.; FRANCO, G. R. Translocação de zinco e crescimento radicular em milho. Revista Brasileira de Ciência do Solo, Viçosa, v. 24, n. 4, p.807-814, 2000.

Cultura Agronômica, Ilha Solteira, v.27, n.2, p.262-272, 2018 
STEINER, F.; ZOZ, T.; JUNIOR, A. S. P.; FRANDOLOSO, J. F.; RUPPENTHAL, V.; JANEGITZ, M. C. Zinco e nitrogênio no desempenho agronômico do milho safrinha. Global Science and Technology, Rio Verde, v. 4, n. 2, p.9-17, 2011.

VALADARES, R. V.; VALADARES, S. V.; FERNANDES, L. A.; SAMPAIO, R. A. Teores de nutrientes no solo e nutrição mineral do milho em áreas irrigadas com água calcária. Revista Caatinga, Mossoró, v. 27, n. 3, p.169-176, 2014. 